\title{
Application of the Probabilistic Dynamic Synthesis Method to Realistic
}

\author{
Structures \\ Andrew M. Brown* \\ NASA Marshall Space Flight Center, Huntsville, AL 35812
}

Aldo A. Ferri ${ }^{+}$

Georgia Institute of Technology, Atlanta, GA 30332

\begin{abstract}
The Probabilistic Dynamic Synthesis method is a technique for obtaining the statistics of a desired response engineering quantity for a structure with non-deterministic parameters. The method uses measured data from modal testing of the structure as the input random variables, rather than more "primitive" quantities like geometry or material variation. This modal information is much more comprehensive and easily measured than the "primitive" information. The probabilistic analysis is carried out using either response surface reliability methods or Monte Carlo simulation. In previous work, the feasibility of the PDS method applied to a simple seven degree-of-freedom spring-mass system was verified. In this paper, extensive issues involved with applying the method to a realistic three-substructure system are examined, and free and forced response analyses are performed. The results from using the method are promising, especially when the lack of alternatives for obtaining quantitative output for probabilistic structures is considered.
\end{abstract}

\section{Nomenclature}

$\beta \quad$ shortest distance from the $\mathrm{g}=\mathrm{O}$ curve to the origin

\footnotetext{
*Aerospace Engineer, Structural Dynamics and Loads Branch, Structural Analysis Division, ED23, MSFC, AL 35812, Member AlAA ${ }^{+}$Associate Professor, Woodruff School of Mechanical Engineering, Georgia Tech, Atlanta, GA 30332, Member AIAA
} 
$[\mathrm{C}]^{\mathrm{a}} \quad$ Correlation matrix of rv's in substructure. a

CDF cumulative distribution function

CMS component mode synthesis

dof's degrees of freedom

$\Phi($.$) \quad Gaussian CDF function$

FORM First Order Reliability Method

$\left[\mathrm{G}_{\mathrm{bb}}\right]^{\mathrm{m}, \mathrm{i}} \quad$ residual flexibility matrix

g limit state function

$\{\lambda\}^{m, i} \quad$ eigenvalues

$[\Phi]^{\mathrm{m}, \mathrm{i}} \quad$ eigenvectors

PDS probabilistic dynamic synthesis

$\mathrm{p}(\mathrm{Y}<\mathrm{y}) \quad$ the probability that $\mathrm{Y}$ is less than $\mathrm{y}$

MC Monte Carlo

rv random variable

$\mathbf{U} \quad$ vector of uncorrelated std. normal rv's

$\{\mathrm{x}\} \quad$ vector of correlated normal rv's

$\{x\}^{\prime} \quad$ vector of correlated std. normal rv's

$\tilde{\mathbf{x}} \quad$ median of $\mathbf{x}$

$\mathrm{x}_{\mathrm{b}} \quad$ boundary dof's

$\mathbf{x}_{\mathrm{i}} \quad$ internal dof's

$\mathbf{x}_{\mathrm{o}} \quad$ internal dof's with applied load

$\mathbf{X}$ vector of random variables

X* design or most probable point (MPP)

$\mathrm{Y}(\mathbf{X}) \quad$ performance function 


\section{Introduction}

Accounting for the statistical geometric and material variability of structures in analysis has been a topic of considerable research for the last 30 years. The determination of quantifiable measures of statistical probability of a desired response variable, such as natural frequency, maximum displacement, or stress, to replace experience-based "safety factors" has been a primary goal of this research. There are several problems associated with its satisfactory application to realistic structures, though. The first problem is accurate definition of the input random variables (rv's). The random variability of the material characteristics is generally available, but the variation in the geometry of a complex structure, such as a hollowed-out turbine blade, is virtually impossible to define accurately. The second problem for analysis is the large size of the finite element models frequently used to simulate these structures. Substructuring reduction techniques are required to decrease the size of many structural systems just to perform a single, deterministic analysis. Finally, a significant problem in the analysis of probabilistic structures is the accurate generation of the Cumulative Distribution Function (CDF) necessary to obtain the probability of the desired response variable. Monte Carlo (MC) simulations can be performed to calculate probability distributions of the desired output, but several thousand runs are required for accurate results.

This research applies a methodology previously developed by the authors, called "probabilistic dynamic synthesis" (PDS) to solve these problems ${ }^{1}$. The PDS method uses dynamic characteristics of substructures measured from modal test as the input random variables rather than "primitive" random variables such as material and geometric variability. These characteristics, which are the free-free eigenvalues, eigenvectors, and residual flexibility, are readily measured and for many substructures, a reasonable sample set of these measurements can be obtained. The variation in these dynamic attributes accurately accounts for the entire random variability of the substructure. This is in contrast to using "primitive" rv's, which no matter how well measured, cannot capture the variation of every 
geometric grid point location in addition to the variation in material properties. Using the residual flexibility method of component mode synthesis (CMS), these dynamic characteristics are used to generate sample models of the substructures that are coupled to form sample system models, which are considerably smaller than an unreduced finite element model. Finally, these sample models are used to obtain the CDF of the response variable by either applying Monte Carlo simulation or by generating datapoints for use in the response surface reliability method, which saves a substantial amount of computer time.

The previous work by the authors verified the feasibility of the PDS method on a simple seven degree-of-freedom (dof) spring-mass system. In this paper, we examine the extensive issues involved with applying the method to a realistically-modeled three substructure system. Both free response and forced response analyses are performed. The goal of the free-response analysis is to quantify a $+/-3 \sigma$ band about the median natural frequency, compared to the somewhat arbitrary $+/-5 \%$ band about the deterministic solution frequently used in industry. The goal of the forced response analysis is to quantify $a+3 \sigma$ value for the maximum response of a dof on the structure, compared with the completely experience-based "factor of safety" presently applied to the maximum responding dof in a deterministic analysis. These results are presented by comparing a CDF obtained using a Monte Carlo simulation of the "baseline" model, in which "primitive" random variables are used, with a CDF using the PDS method. While there is considerable room for improvement, these results are promising, especially when existing options for design of realistic structures are considered. Finally, potential sources of error and avenues of further research are presented.

\section{Probabilistic Background}

The research described in this paper employs the response surface reliability method approach of probabilistic structural mechanics for determining the statistical structural response characteristics. A more extensive review of the reliability methods is presented 
by Brown ${ }^{2}$. To briefly review this technique, consider a limit state function $\mathbf{g}(\mathbf{X})=\mathrm{Y}(\mathbf{X})$ $y$, where $y$ is specific value. Cornell ${ }^{3}$, and Hasover \& Lind $^{4}$ developed the First Order Reliability Method (FORM) by dividing the $\mathbf{X}$ space into two parts, $\mathrm{g}<\mathrm{O}(\mathrm{Y}<\mathrm{y})$ and $\mathrm{g}>$ $\mathrm{O}(\mathrm{Y}>\mathrm{y})$, and approximating $\mathrm{g}$ as a first order Taylor series expanded about the mean of each rv.

If $\mathbf{X}$ is transformed to independent standard normal rv's $\mathbf{U}$, then this first order approximation allows the multi-dimensional probability density function (PDF) to be represented by a one dimensional Gaussian PDF (see Fig. 1). Therefore, $P(Y<y) \approx \Phi(\beta)$, where $\Phi($.$) is the Gaussian CDF function found in handbooks, and \beta$ is shortest distance from the $\mathrm{g}=\mathrm{O}$ curve to the origin, located at the Most Probable Point (MPP). This greatly simplifies the calculation of probability values for a given limit state. The reliability method was expanded by Rackwitz ${ }^{5}$ to multi-dimensional problems for which the limit state curve $g=O$ is an explicit, nonlinear function of the rv's, which makes the determination of $\beta$ much more difficult. The method makes use of Lagrange techniques in an iterative algorithm to find $\beta$. Rackwitz \& Feissler ${ }^{6}$ and Chen $\&$ Lind $^{7}$ continued the development of this method by examining how to develop an "equivalent normal distribution" for rv's with non-normal distributions.

The application of FORM and its extensions for non-explicit limit state functions, as is the case for large structural finite element models, requires the use of numerical differentiation to obtain a first order approximation of the performance function $Y(X)$. A CDF can be constructed from this first order approximation using the technique described above or directly from the first order approximations of the mean and standard deviation if the distribution is assumed to be Gaussian. A practical second order approach was developed in 1987 by Wu \& Wirshing ${ }^{8}$, who improved the accuracy of probability levels obtained by FORM for non-explicit limit states by using a partial second order expansion called the Advanced FORM (AFORM). Initially, the FORM is performed and MPP's obtained. The limit state is then expanded about each MPP using only the first order and 
the pure (no mixed variable terms) second order terms of the Taylor series expansion. The first and second order terms are then "linearized" using a change of variable, and the MPP search algorithm used in FORM is applied. A CDF can also be generated directly from this partial second order approximation if a lognormal distribution is assumed. Wu also developed the Advanced Mean Value (AMV) method, a procedure for updating the FORM or AFORM solution by using the original, exact solution for $\mathrm{g}$. All of these reliability methods have been implemented into a new probabilistic finite-element computer code developed by Southwest Research Institute, NESSUS ${ }^{9}$.

\section{System Description}

In order to examine the applicability of the PDS method for a realistic design problem, a structural system had to be chosen that met several criteria. First, the structure had to be modeled using standard methodology. This was satisfied by using the widely used commercial finite element code, NASTRAN. Next, since tying CMS together with probabilistic methods is an important goal of this research, the size of the model had to be large enough to be able to realize a substantial reduction in computer time due to dynamic reduction. This requirement is satisfied by using a structural system composed of a "disk," which is made up of 630 quad4 plate elements and constrained at the center, and two "blades," which are each composed of 24 quad4 plate elements (see Fig. 2). To pave the way for future mistuned bladed-disk analyses, the disk was assumed to be deterministic and the blades non-deterministic. For ease of analysis, the standard assumption that in-plane translation and rotations are small has been applied. Finally, the structure had to possess a "generic" level of randomness not easily defined by a single variation in a material or geometric property. A single rv would not be able to capture variations in mode shapes that are independent of variations of natural frequency, for instance. To achieve this goal, each blade was separated into three sections, with two of the sections having a thickness set to be an independent normally distributed primitive random variable. In addition, the density of 
each blade across all three sections was defined as a normally distributed independent rv, thus giving each blade three independent rv's (see Table 1).

\section{Monte Carlo Baseline Analysis}

A Monte Carlo analysis of the original, unsubstructured system using the assumed primitive random variable set was chosen to be a baseline for comparison with PDS. A 1000 sample case was executed, taking over 20 hours of wall-clock time. In addition, MC analysis was required to simulate the modal testing phase of the PDS methodology to obtain the statistics of the dynamic rv's of the probabilistic blade substructures. The NESSUS computer code has a new interface with NASTRAN that manages the simulation, both by creating the random vector set for the given input random variables, and by automatically submitting the jobs. The input random variables can be any defined NASTRAN geometric or material property.

\section{Probabilistic Dynamic Synthesis}

The PDS methodology makes use of the residual flexibility method of CMS. This method has been developed by MacNeil ${ }^{10}$, Craig \& Chang ${ }^{11}$, and Martinez et al. ${ }^{12}$. The essential idea in CMS is that substructure modes are truncated since their higher modes will not have a major effect on the combined system modes. The residual flexibility method incorporates the effects of the higher modes by determining their flexibility. A side benefit is that all the elements of the system stiffness matrix can be obtained from test and that the mass matrix can be closely approximated by a unity matrix in the non-boundary partition. Since all the information can be obtained from test, all the probabilistic information can be incorporated into the system matrices for response analysis.

The first step of the probabilistic dynamic synthesis (PDS) method is to divide the model of a structure into substructures $m=a, b, . ., p$, and the degrees of freedom into internal and boundary locations. Each substructure is represented by $\mathbf{n}$ samples, each of which is 
modally tested in a free interface condition. For this research, this testing step is simulated by a MC analysis of the structure using the defined primitive rv's. For substructure $\mathrm{m}$, sample $i$, the test will yield eigenvalues $\{\lambda\}^{\mathrm{m}, \mathrm{i}}$ and eigenvectors $[\Phi]^{\mathrm{m}, \mathrm{i}}$. The eigenvectors have to be consistent from one sample to the next, requiring an interfacing FORTRAN code to assign a consistent sign to the mode shapes for each sample and obtaining a consistent set of rigid-body modes (parallel to the coordinate system axes) for the two free-free modes, which is achieved by using the SUPORT card with the modified Householder method in NASTRAN. In addition, the boundary partition of the residual flexibility matrix $\left[G_{b b}\right]^{m, i}$ is obtained from the measured boundary drive point frequency response functions of the boundary coordinates ${ }^{13}$. Here, the residual flexibility is calculated analytically by subtracting the flexibility for the retained modes from the total system flexibility (inverse of the stiffness matrix) for the constrained substructure. For the free-free substructures, the system is unconstrained so the stiffness matrix is singular. The "inertia-relief method," initially developed by $\mathrm{Craig}^{14}$, is therefore used to obtain the residual flexibility.

For the free-response solution using the PDS method, only the kept (non-truncated) eigenvalues, the boundary coordinates of the kept eigenvectors, and the boundary partition of the residual flexibility matrix are needed. These values can be combined into a single vector $\{x\}^{m, i}$, defined as

$$
\{x\}^{\mathrm{m}, \mathrm{i}}=\left[\lambda_{1} \ldots \lambda_{\mathrm{k}}\left\{\phi_{\mathrm{b}}\right\}_{1}^{\mathrm{T}} \ldots\left\{\phi_{\mathrm{b}}\right\}_{\mathrm{k}}^{\mathrm{T}} \mathrm{G}_{11} \ldots \mathrm{G}_{\mathrm{bb}}\right]^{\mathrm{m}, \mathrm{i} \mathrm{T}}
$$

where $\mathrm{k}$ is the number of kept modes. If the entire sample of substructure $\mathrm{m}$ is tested, $\{\mathrm{x}\}^{\mathrm{m}}$ can therefore be defined as a vector composed of elements that are each a random variable with measured mean and standard deviation. This vector is now transformed to $\left\{\mathrm{x}^{\prime}\right\}^{\mathrm{m}}, \mathrm{a}$ vector of standard normally distributed rv's, using the measured mean and standard deviation of each rv. An important assumption is made that the original rv's in $\{\mathbf{x}\}$ are normally distributed. This distribution is required for decorrelation of the random variables (discussed below), and is somewhat accurate, as shown in distribution-matching 
tests published in the previous work by the authors ${ }^{1}$. If the measured distribution were found to match another standard type better, then an could be obtained. Since the match with a Gaussian distribution is not perfect, though, there is some error introduced. A technique to address this error will be discussed later in this paper. Another method of increasing the accuracy would be to obtain an "equivalent normal" distribution using using the Chen-Lind three-parameter method ${ }^{7} \quad$ In addition, there will be some degree of correlation between each of the random variables, which can be calculated from the measured data. This information is placed in a correlation matrix $[\mathrm{C}]^{\mathrm{m}}$ relating each element with every other element. For the FORM analysis, a set of independent random variables $\{\mathrm{u}\}^{\mathrm{m}}$ is required. This can be accomplished by making an orthogonal transformation of $\left\{x^{\prime}\right\}^{\mathrm{m}}$ with the eigenvectors of the correlation matrix to uncouple the $\left\{\mathrm{x}^{\prime}\right\}$ coordinates, thereby creating $\{\mathrm{u}\}^{\mathrm{m}}$. This can be expressed for substructures $\mathrm{m}=\mathrm{a}, \mathrm{b}, . ., \mathrm{p}$ as

$$
\left\{x^{\prime}\right\}^{m}=[\Phi]_{C}^{m}\{u\}^{m}
$$

It becomes evident at this time that the size of the dynamic rv set is intractable for a realistic problem. For this case, which has only two probabilistic substructures and where 20 modes are retained (out of a possible 108) per substructure, the number of dynamic rv's is 802 . Several assumptions are therefore made to drastically reduce the number of dynamic rv's. The first is to assume that the limit state function is insensitive to the variation in the rotational dof's in the modes. This reduces the size of eigenvector rv's from 240 to 80 per substructure. The second is to assume the limit state is insensitive to not only the rotational dof's, but also to the off-diagonal terms in the boundary residual flexibility matrix, which reduces the size of that contribution from 144 to 4 per substructure. These assumptions do not remove these variables from the formulation of the substructure stiffness matrices; instead, it allows the use of the mean value of those variables (or median, as will be discussed later). Admire, et. $\mathrm{al}^{15}$ examined the effect of completely removing the 
off-diagonal $\mathrm{G}$ elements, and calculated a natural frequency error of less than $5 \%$ between the exact value and the value obtained using the residual flexibility formulation.

In addition, a substantial reduction in the number of independent rv's is achieved by eliminating "artificial" rv's. These were first found by calculating the correlation matrix of the independent rv's $[\mathrm{C}]_{\mathrm{u}}$. Since the elements of $[\mathrm{C}]_{u}$ are uncorrelated, it was expected to be an identity matrix. The result was somewhat different; for the rows/columns of $[\mathrm{C}]_{u}$ that corresponded to eigenvalues on the order of one, the diagonal term was equal to 1.0 and the other elements in the associated row/column were very small. However, many of the eigenvalues turned out to be very small and the analogous rows and columns in $[C]_{u}$ were not diagonal; the diagonal values were 1.0 but the other terms in the row/column were all on the order of one (between zero and one) instead of being close to zero. This can be explained by realizing that the actual number of independent rv's in the system is equal to the original number of independent primitive rv's, not the number of dynamic rv's. Therefore, the rv's associated with the small $\lambda_{\mathrm{C}}$ values are actually insignificant and the magnitudes of these values are mainly due to numerical roundoff. The high off-diagonal values in the new correlation matrix in these rows/columns therefore indicates a large amount of correlation between these numerical artifact "rv's". The above result can be used to further decrease the size of the rv set $\{u\}$. A sum of the eigenvalues of the original correlation matrix is calculated, and any eigenvalue less than $3 \%$ of the sum is deemed insignificant and the associated rv in $\{u\}$ neglected. The value of $3 \%$ was reached by starting at 5\% and decreasing the cutoff value (thereby including more rv's) until the final result stabilized. This reduced the eigenvalue set for each blade from 101 to 11 . It is unclear why this value did not actually reduce to three, which is the number of primitive rv's per blade, but nevertheless, this reduction greatly facilitated the analysis.

To generate a first order Taylor series representation of the limit state, each independent random variable is varied individually by some percentage of its standard deviation $\sigma$, which is equal to the square root of the corresponding eigenvalue of the 

correlation matrix, while the other rv's are kept constant at their mean values. For higher order methods, each independent rv is varied by two different amounts to allow a quadratic curve fit. Matrix $[\mathrm{u}]$ is created by varying each of its elements by $+/-0.3$ of a standard deviation, which for that rv is the square root of the correlation matrix eigenvalue. The number of rows in $[u]$ is equal to the number of probabilistic substructures times the reduced number of independent rv's, and the number of columns is equal to this number times two plus one additional column for the mean value set, as shown below. The superscripts on the rv $u$ refer to the number of the probabilistic substructure, and the subscript refers to the number of the independent rv.

$$
[u]=\left[\begin{array}{cccccc}
-.3 \sigma_{u_{1}^{1}} & .3 \sigma_{u_{1}^{3}} & 0 & 0 & 0 & 0 \\
0 & 0 & \ddots & 0 & 0 & 0 \\
0 & 0 & 0 & -.3 \sigma_{u_{1}^{j}} & .3 \sigma_{u_{1}^{j}} & 0
\end{array}\right]
$$

Each column in $[\mathrm{u}]$ is then transformed to the set of correlated standard normal rv's $\{\mathrm{x}\}^{\prime}$ using the transpose of $[\Phi]_{C}$, and then into the original rv's $\{x\}$ using the standard normal transformation. The new vectors $\{\lambda\},[\Phi]$, and $\left[\mathrm{G}_{\mathrm{bb}}\right]$ are pulled out from $\{\mathrm{x}\}$ and placed in substructure mass and stiffness matrices according to the residual flexibility formulation:

$$
\mathrm{K}^{\alpha}=\left[\begin{array}{cc}
\Lambda^{\mathrm{k}}+\boldsymbol{\Phi}_{\mathrm{b}}^{\mathrm{kT}} \mathrm{G}_{\mathrm{bb}}^{-1} \boldsymbol{\Phi}_{\mathrm{bk}} & -\boldsymbol{\Phi}_{\mathrm{b}}^{\mathrm{kT}} \mathrm{G}_{\mathrm{bb}}^{-1} \\
\operatorname{sym} & \mathrm{G}_{\mathrm{bb}}^{-1}
\end{array}\right], \quad \mathrm{M}=\left[\begin{array}{cc}
\mathrm{I} & 0 \\
0 & 0
\end{array}\right]
$$

where $\Lambda^{\mathrm{k}}$ is a diagonal matrix of the kept eigenvalues $\{\lambda\}^{\mathrm{k}}$. For the purposes of this study, the third natural frequency of the combined system was selected as the response value of interest, so the system matrix is then compiled and an eigensolution performed to obtain this value for each column of $[u]$. The columns of $[u]$ along with the associated response value are then input into the NESSUS code where the AFORM partial quadratic method can be applied. By simply changing the first variation in the above approximation to a value very close to zero times the standard deviation, the same program can also be easily altered to create a FORM linear approximation. 
After initial examination of the results showed a poor comparison with the MC baseline, an important subtlety was found in the application of the transformation to standard normal variables. For the MC baseline solution, the results are obtained by creating a normal distribution about the mean values of the primitive rv's; this distribution is symmetric, so the median is equal to the mean. Since the finite element solution is slightly nonlinear as a function of the input rv's, though, the mean of the solution will not equal the median, but the median solution will result from using the mean (equal to median) primitive rv's.

As PDS is implemented for these analytical simulations, though, there is an intermediate step that introduces error. Symmetric Gaussian MC distributions are created about the means of the dynamic rv's, but these rv's are actually not purely Gaussian, since a slightly nonlinear eigensolution has been performed to obtain them, and the means will not equal the medians. This results in a skew of the entire CDF curve since the method assumes that the dynamic rv's do in fact follow a symmetric distribution. The error can be substantially corrected by simply ensuring that the median primitive values are carried through the procedure to generate the median results. This is accomplished by using the medians $\tilde{\mathrm{x}}$ of the calculated dynamic rv's as the "mean" in the transformation to standard normal coordinates:

$$
(\mathrm{x})=\left\{\mathrm{x}^{\prime}\right\} \sigma+\tilde{\mathrm{x}}
$$

This change drastically improved the results in both the mean and standard deviation of the results since it moves the expansion of the limit state about a more accurate location in the u-space.

A parallel application of the PDS method is to apply the dynamic rv's using the MC approach rather than the reliability approach. The MC should provide more accurate answers, while the reliability method will be more computationally efficient. This technique 
is performed by using the statistical data obtained using the modal testing simulation to create normally distributed samples for each column in [u] rather than creating "load cases" for the Taylor series. This will result in a [u] matrix with the number of columns equal to the number of Monte Carlo samples.

\section{Results}

The PDS FORM and PDS MC results for the CDF of the system third natural frequency are compared with the MC baseline in Figure 3 and Table 2. This frequency was chosen because the associated two nodal diameter mode shape is similar to the type of excitation shape found in turbomachinery which the forced response analysis would simulate. The higher order reliability methods were also applied to this problem, but they did not show any improvement over the linear case; this may be due to the very small nonlinearity of the limit state. From a design problem approach, the errors as seen on the table for the .01 and $.99 \mathrm{CDF}$ levels are less than $2 \%$, so the values could be used with confidence. From a theoretical standpoint, the curves do not line up extremely well; this is indicated by the error in the standard deviation of about $20 \%$. Potential sources of error include the following: 1) transformation error of the statistics of the original primitive rv's due to inaccuracy of the MC simulation of the dynamic rv's (possibly insufficient number of samples), 2) transformation error in the statistics and recorrelation of the rv's due to their assumed normality, 3) error due to truncation of the number of independent rv's, 4) a small truncation error due to using the RF formulation of CMS (examined for the deterministic case), and 5) programming error.

It is interesting to compare computational times at this point. All the analyses were performed on a CRAY T-90 supercomputer. The MC baseline analysis took 2527 CPU seconds and 20.3 hours of wallclock time to run. PDS MC took 389.5 seconds of CPU time and 1112.3 seconds (18.5 minutes) wallclock time, a drastic reduction enabled by the continuous generation, storage, and analysis of modally reduced system matrices rather 
than the complete model generation and analysis necessary for each of the MC baseline samples. The FORM method took only 20.1 seconds of CPU and 64.2 seconds of wallclock to create the datapoints for input to NESSUS, and about 30 seconds wallclock to run NESSUS to perform the FORM solution. This further reduction is due to the substantial decrease in the number of solutions generated. As anticipated, both the use of CMS and probabilistic methods drastically decreased the amount of time necessary to run the analysis, which, especially for a more detailed model, could be a pre-requisite for use in design.

\section{Forced Response}

The use of PDS was now expanded to forced response. In particular, a frequency response solution of the system was derived since this type of excitation would prove most applicable to the bladed-disk problem. For forced response, the dof's of a substructure are partitioned into three sections, $\mathbf{x}_{0}$, which are internal dof's with no external load applied, $\mathbf{x}_{\mathbf{i}}$, internal dof's with an external load applied, and $x_{b}$, boundary dof's which may or may not have external load applied. The residual flexibility transformation matrix from the original set of coordinate to the generalized set is therefore:

$$
\left\{\begin{array}{c}
x_{0} \\
x_{i} \\
x_{b}
\end{array}\right\}=\left[\begin{array}{ccc}
\Phi_{i}-G_{\text {res }-a b} G_{\text {res }-b b}^{-1} \Phi_{b} & 0 & G_{\text {res }-o b} G_{\text {res }-b b}^{-1} \\
0 & \Phi_{i}-G_{\text {res }-i b} G_{\text {res }-b b}^{-1} \Phi_{b} & G_{\text {res }-i b} G_{\text {res }-b b}^{-1} \\
0 & 0 & \text { I }
\end{array}\right]\left\{\begin{array}{l}
q_{o} \\
q_{i} \\
x_{b}
\end{array}\right\}=[T]\{q\}
$$

The transformed mass and stiffness matrices will be identical to those obtained for the free response shown in Equations (4). To determine the right-hand side of the forced response equation of motion, the transformation matrix and the original load vector are both carried throughout the analysis until the generalized force is needed, at which time the matrix multiplication is carried out. Therefore, in addition to the boundary partitions of the modal matrix and the residual flexibility matrix required for the free response solution, the partitions associated with the dof's with load applied are required for the forced response 
analysis. To more easily perform this analysis, the transformed coupled system is transformed to an uncoupled system using the set of truncated mass-normalized system eigenvectors $[\Phi]_{1}$ resulting from the free response solution of the system, as shown below:

$$
\{q\}_{1}=[\Phi]_{1}\{q]_{2}
$$

The resulting uncoupled system is in the form

$$
[\mathrm{I}]\{\ddot{\mathrm{q}}\}_{2}+[\mathrm{C}]\{\dot{\mathrm{q}}\}_{2}+[\Lambda]_{1}\{\mathrm{q}\}_{2}=[\boldsymbol{\Phi}]_{1}^{\mathrm{T}}[\mathrm{T}]^{\mathrm{T}}\{\mathbf{f}\}
$$

where $[\Lambda]_{1}$ is the diagonal matrix of the eigenvalues of the transformed system. The standard (viscous) assumption of constant modal damping of $0.5 \%$ is used for [C]. The input force is a system of four harmonic loads, each of amplitude $1 \mathrm{lb} .(4.448 \mathrm{~N})$ and applied in-phase with a peak displacement of the two nodal diameter mode shape to simulate bladed-disk excitation. There are standard techniques available for obtaining the frequency response solution for this set of uncoupled single-dof systems. Because of the series of linear transformations performed on the solution, though, a complex quantity for the solution is desired rather than one in terms of magnitude and phase, as is commonly derived in texts on the subject and which cannot be easily transformed. This complex vector $\{\mathrm{q}\}_{2}$ can now be transformed back to the original coordinates $\{x\}$ using equations $(6)$ and $(7)$ :

$$
\{\mathrm{x}\}=[\mathrm{T}][\Phi]_{1}\{\mathrm{q}\}_{2}
$$

The absolute values of this complex vector are then calculated to obtain the physical response values.

As seen above, applying PDS for the forced response solution requires more information from the modal data and the residual flexibility matrices. This increases the number of dynamic rv's necessary to solve the problem, which increases the complexity of the problem in several aspects. To minimize this number, it is important to decide a-priori which internal dof's will either have external load applied or require a displacement recovery. Although the entire modal matrix and residual flexibility matrix are calculated, only those 
partitions of the modal matrix and the residual flexibility matrix, along with the boundary dof's, are stored. In addition, only the correlations with these additional dof's are generated.

To address the design problem defined earlier, the response variable for the forced response analysis was chosen to be the maximum response for all dof's in the structure. Initially, the excitation was applied at only a single frequency, but this was changed to a wider excitation bandwidth since the maximum response occurs at the damped natural frequency of the mode shape being excited, and this mode shape can occur over a range of frequencies for a probabilistic structure. It is assumed that the actual excitation mechanism could also vary in frequency by this amount, which is generally the case in engine turbomachinery, for instance. Using the maximum over some frequency range also helps to reduce the extreme variability in response that can be observed at any particular excitation frequency ${ }^{16}$, which would introduce substantial nonlinearities in the response limit state surface. A range of $+/-10 \mathrm{hz}$ about the deterministic natural frequency of $458 \mathrm{hz}$ was used in this case. This value is probably inadequate, since the free response case shows that the actual range is between $438 \mathrm{hz}$ and $472 \mathrm{hz}$, so this may be a source of error in the final results.

The Monte Carlo baseline frequency response solution required extensive alteration of the MSCPOST subroutine within the NESSUS code. The PDS procedure using both the MC and reliability approaches for the frequency response problem is similar to the free response problem. The main additions are the subroutines necessary for calculation of the different $[\mathrm{T}]$ matrices for each substructure and the solution algorithm for the complex frequency response. Because the value of the largest responding dof is no longer at a frequency known a-priori (since the third natural frequency and third natural mode will vary for each statistical sample), a sorting algorithm to find the maximum value was used that scanned the response values for all the selected dof's for all the frequencies in the bandwidth chosen. The dof's selected were those at the blade tips, which were assumed to contain the maximum responding dof of the entire structure for the chosen excitation. This maximum 
displacement value was then used as the NESSUS response variable, and input along with the corresponding load set. PDS MC and FORM cases were run as well as a normal distribution using the linear approximate mean and standard deviation. The PDS quadratic AFORM method using the medians was also attempted, but the AFORM algorithm in NESSUS/FPI never converged to a solution, which is a problem that sometimes occurs with the technique ${ }^{17}$.

The CDF results for the PDS methods are plotted along with the 1000 sample MC baseline simulation in Figure 4 and Table 3 . The MC baseline CDF is extremely unsymmetric, so this clearly causes a problem for the reliability methods. This asymmetry may be due to the fact that the maximum response is obtained by scanning the response of different nodes, and this may skew the distribution. Further examination of this phenomena was performed on a more detailed bladed-disk, which will be reported in the future, and additional work is also needed. It is also noted that there is fair agreement between the MC baseline result and that obtained from MC using dynamic rv's. The errors for the design point at .99 are still around $10 \%$ for both PDS methods, though, which is within reason especially when one considers that at present, there are no alternative methods for obtaining this maximum value. As with the free-response analysis, the significant error in the standard deviation and the lack of coincidence of the curves indicated visually signifies that there is still error present in the methods. An additional source of error in the reliability methods for this analysis is the extent to which the maximum responding dof does not have a smooth dependence on the rv's. Possible discontinuities in this dependence would cause errors in the response surface generation created using numerical differentiation. An examination of the run times yields similar results as the free response case, with the MC baseline taking 27.3 hours of wall-clock time, the PDS MC taking 18.5 minutes wall-clock, and the PDS FORM taking about 90 seconds. Because of the acceptable level of error for design, the increased accuracy in system variability obtained by using dynamic rather than 
primitive rv's, and the tremendous reduction in computer time, the technique was applied to the solution of a mistuned bladed-disk, which will be reported in a future paper.

\section{Conclusions}

A probabilistic dynamic synthesis method has been applied to the analysis of a realistic three-substructure system. The new technique uses modal data from a sample set of substructures to generate a set of dynamic random variables which fully describe the probabilistic variation in the structures. The residual flexibility method of component mode synthesis is then used to generate probabilistic mass and stiffness matrices which can be used to obtain any desired response variable. Probabilistic analysis is performed on these stochastic systems by applying both the traditional Monte Carlo technique and new

reliability methods. Solutions for both free and forced response of the realistic system were obtained, and the results compare favorably with a baseline Monte Carlo analysis. The new method results in a dramatic decrease in computer run-time as well as increased accuracy in representation of the probabilistic variation of the parameters of the structural system. 


\section{References}

${ }^{1}$ Brown, A.M. and Ferrri, A.A., "Probabilistic Component Mode Synthesis of Nondeterministic Substructures," AIAA Jouirnal, Vol. 34, No.4, 1996, pp. 830-834.

${ }^{2}$ Brown, A.M., "Development of a Probabilistic Dynamic Synthesis Method for the Analysis of Non-Determinististic Structures," Ph.D. Dissertation, Georgia Institute of Technology, 1998, pp. 18-51.

${ }^{3}$ Cornell, C.A., "A Probability-Based Structural Code," Journal of the American Concrete Institute, Vol. 66, No. 12, December 1969, pp. 974-985.

${ }^{4}$ Hasofer, A.M. and Lind, N.C., "Exact and Invariant Second Moment Code Format," Journal of the Engineering Mechanics Division, ASCE, Vol. 100, No. EM1, 1974, pp.111-121.

${ }^{5}$ Rackwitz, R., "Practical Probabilistic Approach to Design," Bulletin No. 112, Comite Europen du Beton. Paris, France, 1976.

${ }^{6}$ Rackwitz, R. and Fiessler, B., "Structural Reliability Under Combined Random Load Sequences," Computer and Structures, Vol. 9, No. 5, 1978, pp. 489-494.

${ }^{7}$ Chen, X. and Lind, N.C. , "Fast Probability Integration by Three-Parameter Normal Tail Approximation," Structural Safety, Vol.1, 1983, pp. 269-276.

${ }^{8}$ Wu, Y-T., and Wirsching, P., "New Algorithm for Structural Reliability Estimation," Journal of Engineering Mechanics, ASCE, Vol. 113, No. 9, September, 1987, pp. 1319-1336.

${ }^{9}$ Wu, Y.T., "FPI Theoretical Manual, NESSUS Reference Manual," Southwest Research Institute, Version 1.0, 1991.

${ }^{10}$ MacNeal, R.H., "A Hybrid Method of Component Mode Synthesis," Computers and Structures, Vol. 1, 1971, pp. 581-601.

${ }^{11}$ Craig, R.R. and Chang, C., "Free-Interface Methods of Substructure Coupling for Dynamic Analysis," AIAA Journal, Vol. 14, No. 11, 1976, pp.1633-1635.

${ }^{12}$ Martinez, D.R., Carne, T.G. and Miller, A.K., "Combined Experimental/Analytical Modeling Using Component Mode Synthesis," Proceeding of the 25th Structures, Structural Dynamics and Materials Conference, May 1984, pp. 140-152.

${ }^{13}$ Bookout, P., "Statistically Generated Weighted Curve Fit of Residual Functions for Modal Analysis of Structures," NASA TM 108481, 1995.

${ }^{14}$ Craig, R., "Structural Dynamics; An Introduction to Computer Methods.," New York: John Wiley and Sons, 1981. 
${ }^{15}$ Admire, J.R., Tinker, M.L. and Ivey, E.W., "Residual Flexibility Test Method for Verification of Constrained Structural Models," AIAA Journal, Vol. 32, No. 1, 1994, pp. 170-175.

${ }^{16}$ Ginsberg, J.H., and Pham, H., "Forced response of a continuous harmonic system displaying eigenvalue veering phenomena," ASME Journal of Vibration and Acoustics, Vol. 117, 1995, pp. 439-444.

${ }^{17}$ Wu, Y.T., "FPI Theoretical Manual, NESSUS Reference Manual," Southwest Research Institute, Version 1.0, 1991, p. 37. 
"Application of a Probabilitic Dynamic Synthesis Method ...", A. M. Brown, Paper \# J24252

Figure Captions Page

Fig. 1 Joint probability density surface

Fig. 2 3-Substructure system

Fig. 3 3-Substructure mode 3 natural frequency comparing Monte Carlo with PDS

Fig. 4 Percent errors for forced response solution 


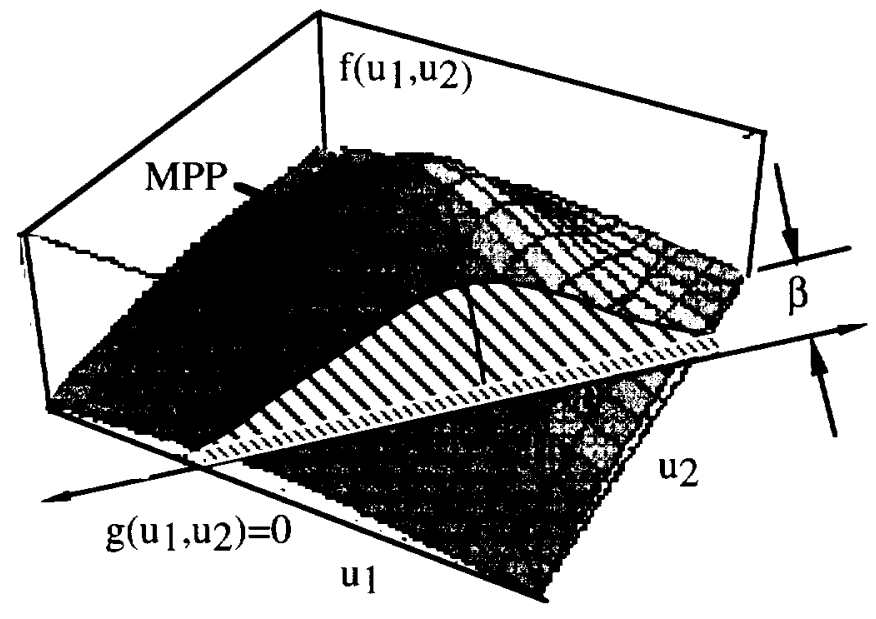




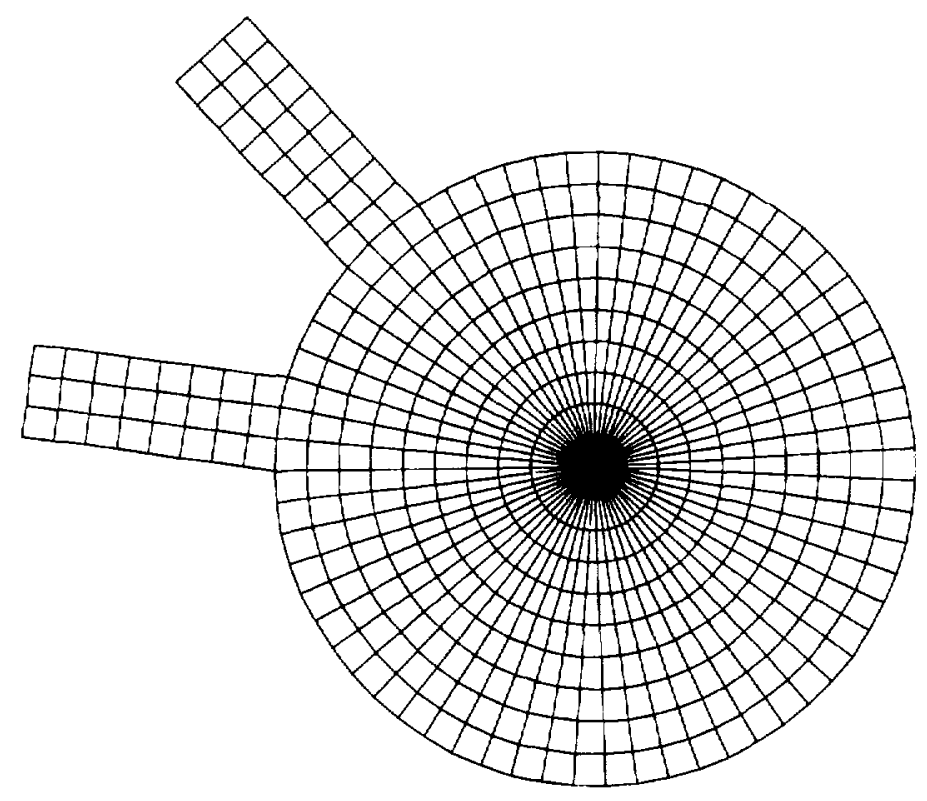




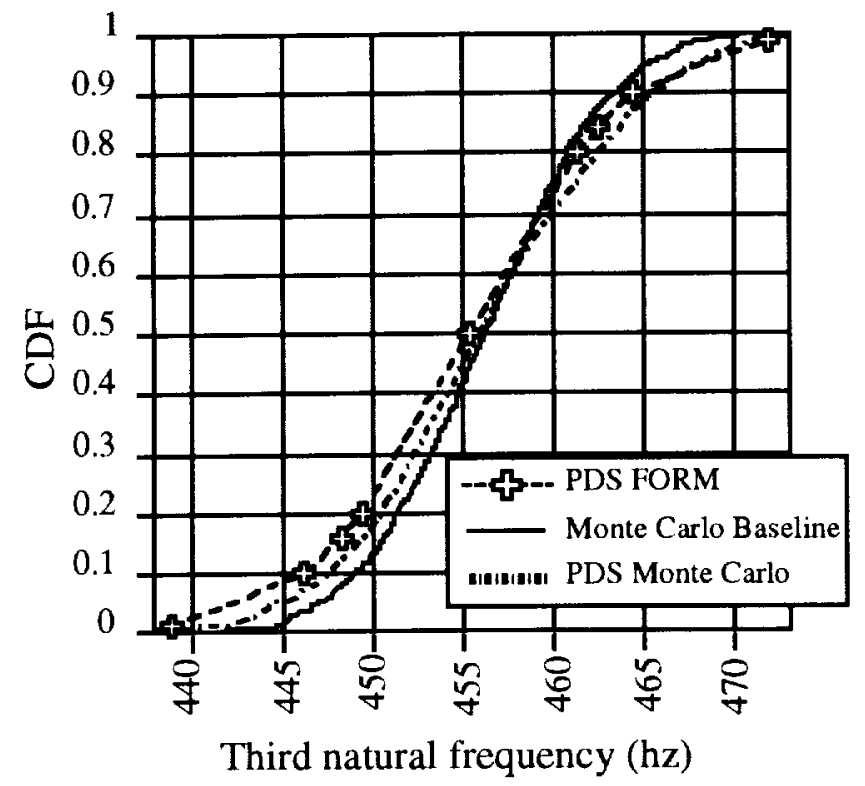




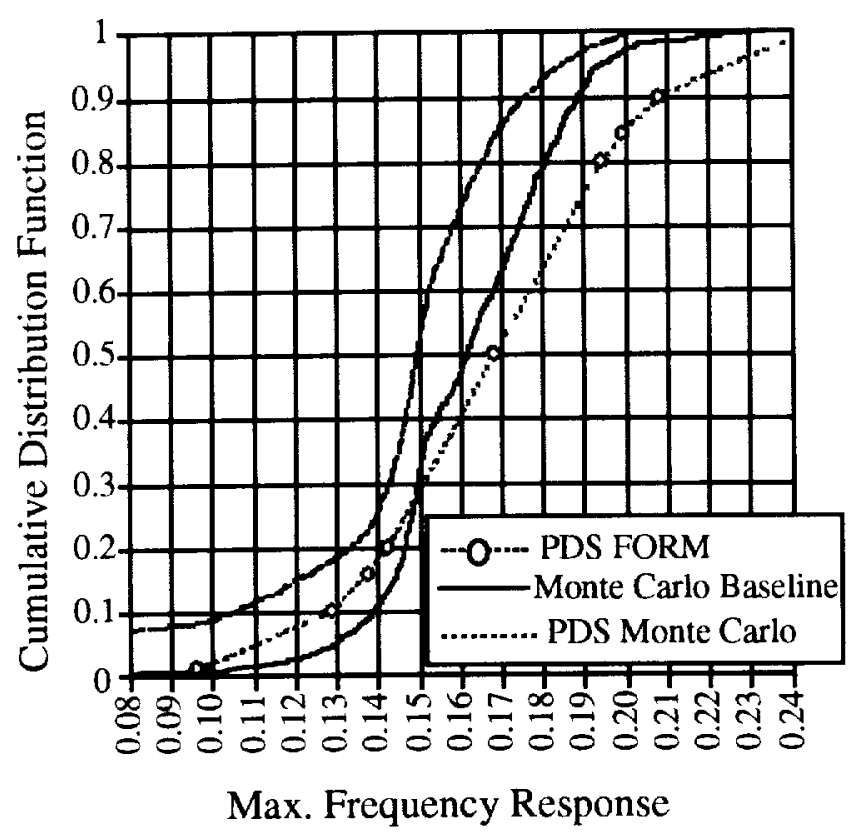


Table 1 3-Substructure System Information

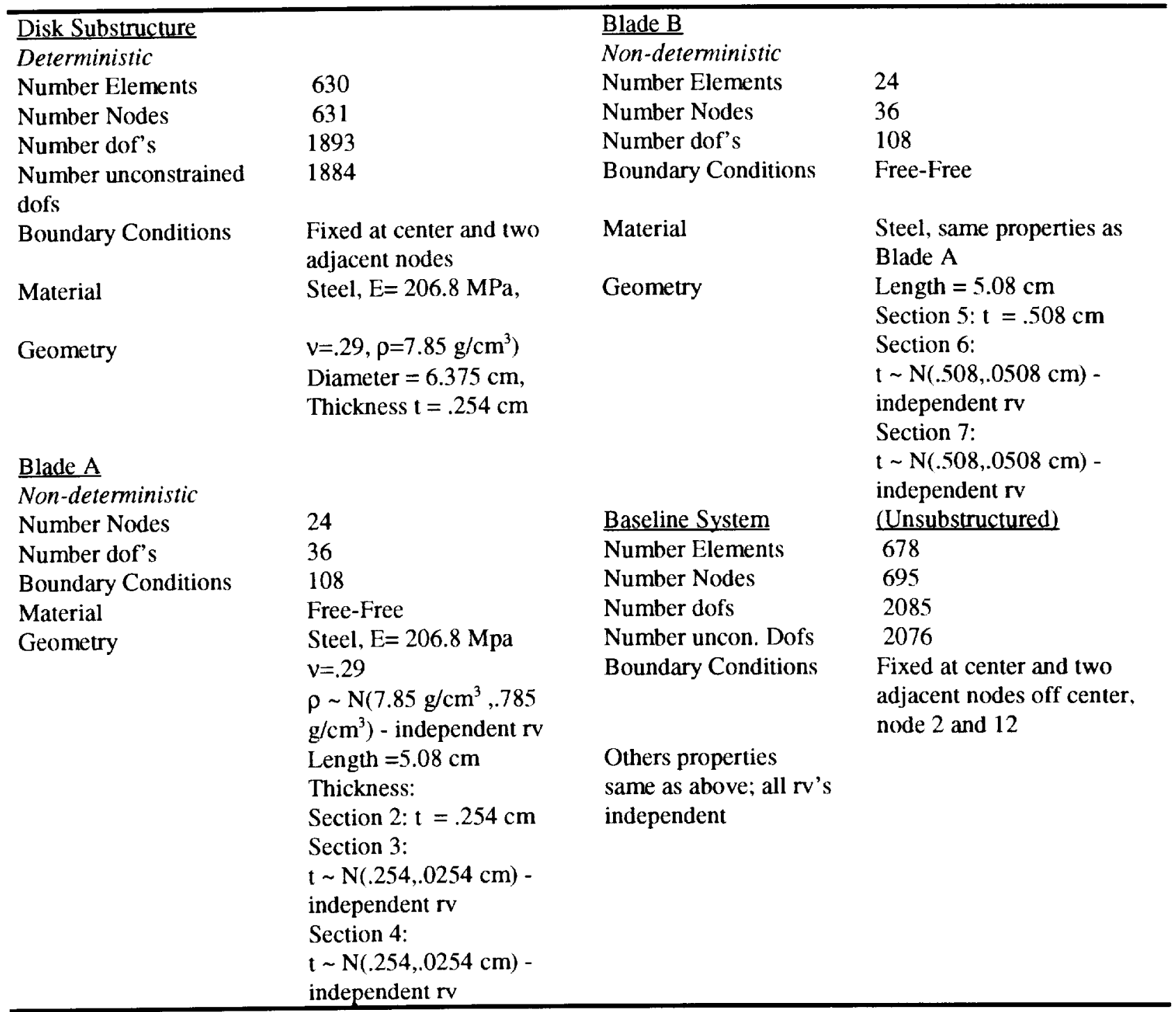


Table 2 Percent Errors for Free Response Solution

\begin{tabular}{lll}
\hline \hline Parameter & PDS form (\%) & PDS Monte Carlo(\%) \\
\hline Median & 0.18 & 0.03 \\
Std. Dev. & 28.91 & 19.97 \\
.01CDF Value & -1.3 & 1.36 \\
.99 CDF Value & 0.69 & -1.79 \\
\hline \hline
\end{tabular}


Table 3 Percent Errors for Forced Response Solution

\begin{tabular}{lll}
\hline \hline Parameter & PDS form (\%) & PDS Monte Carlo(\%) \\
\hline Median & 5.23 & -6.37 \\
Sid. Dev. & 24.33 & 25.06 \\
99 CDF Value & 11.53 & 9.56 \\
\hline \hline
\end{tabular}

